\title{
Inhibition of LCMR1 and ATG12 by demethylation-activated miR-570-3p is involved in the anti-metastasis effects of metformin on human osteosarcoma
}

\author{
Xing Bao ${ }^{1}$, Libo Zhao ${ }^{1}$, Hanfeng Guan ${ }^{1}$ and Feng $\mathrm{Li}^{1}$
}

\begin{abstract}
Epidemiological studies have demonstrated that metformin could mitigate the progression of several tumors. Although it has been proved that metformin could cause demethylation of DNA and lead to up-regulation of some encoding genes and non-coding RNAs, there is little data about the effects of metformin on metastasis, and the interaction between metastasis and autophagy in human osteosarcoma cells. Here, we found miR-570-3p was significantly down-regulated in human metastatic osteosarcoma tissues but not in non-metastatic osteosarcoma tissues. Metformin attenuates the metastasis and autophagy in osteosarcoma. Interestingly, this autophagy favors osteosarcoma cells invasion. Moreover, reduction of metformin-induced inhibition of autophagy could reverse the invasion suppression in osteosarcoma. Mechanistically, metformin increases miR-570-3p by the demethylation of DNA, and the upregulation of miR-570-3p repressed the translation of its target, LCMR1 and ATG12. Our results, for the first time, presents evidence that the miR-570-3p-mediated suppression of LCMR1 and ATG12 is involved in the metformininduced inhibition of metastasis in osteosarcoma cells.
\end{abstract}

\section{Introduction}

Osteosarcoma (OS) is the most common primary malignant bone tumor, which is characterised by an awfully high aggressiveness with rapid development of distant metastasis ${ }^{1-3}$. Current practices for the treatment of osteosarcoma include wide-margin surgical resection and chemotherapy; nearly $30-40 \%$ of patients fail to respond to chemotherapy, typically due to the emergence of metastasis ${ }^{4,5}$. The mechanisms underlying metastasis remain vague. A further investigation of molecular markers that predict metastasis of osteosarcoma is urgent.

Correspondence: Feng Li (lifengmdhust@163.com)

${ }^{1}$ Department of Orthopedics, Tongji Hospital of Tongji Medical College,

Huazhong University of Science and Technology, 1095\#, Jiefang Ave, Wuhan

430030, People's Republic of China

These authors contributed equally: Xing Bao, Libo Zhao.

Edited by B. Zhivotovsky
Increasing evidence reveals that microRNAs (miRNAs) play critical roles in regulating migration and invasion in tumor cells ${ }^{6-8}$. MiRNAs, which are small, noncoding RNA molecules of 20-25 nucleotides, have the capacity to suppress at the post-transcriptional and/or transcriptional level primarily by targeting the 3 '-untranslated regions of mRNAs ${ }^{9,10}$. Aberrant expression of miRNAs have been found in many types of tumors ${ }^{11-15}$. Dysregulation of miRNAs can influence metastasis, chemosensitivity, and tumor cell growth and proliferation ${ }^{16-20}$. It has been reported that several mechanisms result to miRNA dysregulation, including aberrant DNA methylation of CpG islands ${ }^{21,22}$.

Metformin(Met), a relatively inexpensive and well tolerated oral anti-diabetic drug has raised extensive attention for its potential effects in tumor prevention and treatment ${ }^{23,24}$. An increasing number of studies have

\section{(c) The Author(s) 2018}

(c) (i) Open Access This article is licensed under a Creative Commons Attribution 4.0 International License, which permits use, sharing, adaptation, distribution and reproduction c. in any medium or format, as long as you give appropriate credit to the original author(s) and the source, provide a link to the Creative Commons license, and indicate if changes were made. The images or other third party material in this article are included in the article's Creative Commons license, unless indicated otherwise in a credit line to the material. If material is not included in the article's Creative Commons license and your intended use is not permitted by statutory regulation or exceeds the permitted use, you will need to obtain permission directly from the copyright holder. To view a copy of this license, visit http://creativecommons.org/licenses/by/4.0/. 
demonstrated varied mechanisms underlying the antitumor effect of metformin, with disparate mechanisms playing crucial parts in different tissues and in various tumors. It has been proved that metformin could cause demethylation of DNA and lead to up-regulation of some encoding genes and non-coding $\mathrm{RNAs}^{25,26}$. Many studies have shown that metformin could suppress the growth of tumor cells by leading to apoptosis and autophagy $^{27}$.

Currently, there is little data about the effects of metformin on metastasis, and the interaction between metastasis and autophagy in human osteosarcoma cells. Understanding the interplay between metastasis and autophagy modulating by metformin may reveal new targets for tumor treatment. The influence of metformin on human osteosarcoma have not been sufficiently studied and, especially, the antitumor mechanisms of metformin in osteosarcoma have not been previously explored.

Here, we found that metformin weaken the migratory and invasive capacities of osteosarcoma cells in vitro and in vivo. Mechanistically, metformin increases miR-570-3p by the demethylation of DNA, and the increase of miR570-3p repressed the translation of its target, lung cancer metastasis-related protein (LCMR1), which has an important role in tumor metastasis, and another target, autophagy-related gene 12 (ATG12), which is an autophagy marker that is significant for the resistance to apoptosis in many types of tumors.

\section{Result}

\section{Metformin inhibits the migration and invasion of} osteosarcoma cells

Metformin did not markedly affect the vitality of MG63, U2OS or $143 \mathrm{~B}$ cells at concentrations of 2,5 or $10 \mathrm{mM}$ (Fig. 1a). MG63,U2OS and 143B cells displayed high migratory and invasive capacities, although Metformin suppressed migration, as detected by wound healing assays, at a concentration of $10 \mathrm{mM}$ (Fig. 1b). This concentration was selected as the maximum for further studies. Furthermore, as assessed with transwell assays, Metformin attenuated the invasion of osteosarcoma cells (Fig. 1c). These data reveal that metformin could efficaciously weaken the migration and invasion of human osteosarcoma cells.

Invasion of tumor cell is a trademark of metastasis, and that is a complicated process involving cytoskeletal rearrangement, lamellipodia forming, membrane ruffling and cell morphological alters. Cytoskeletal assay of $143 \mathrm{~B}$ and U2OS cells was visualized by confocal microscopy. As demonstrated in Fig. 1d, conspicuous lamellipodial protrusions were formed in the submembranous area of control cells, but the reversed phenomenon was observed in metformin-treated cells, which displayed reconstructed cytoskeleton and well distributed F-actin in cells. This suggests that the treatment of Metformin could significantly affect cytoskeletal rearrangement in osteosarcoma cells.

\section{Metformin-induced inhibition of autophagy indirectly suppresses osteosarcoma cell invasion}

Autophagy-associated cell death is another type of programmed cell death, which has emerged as an important regulator of the migration and invasion in different kinds of tumors ${ }^{28}$. In some metastatic tumors, high level of autophagy before treatment predicts invasiveness, low sensitivity to chemotherapy and poor survival $^{29}$.

To figure out whether metformin is involved in autophagy, transmission electron microscopy (TEM) was carried out to observe the ultrastructures present during autophagy. Osteosarcoma cells treated with metformin and those treated with 3MA exhibited few autophagic vacuoles compared with typical autophagic vacuoles, and a distinct double membrane was present in the control group (Fig. 2a).

LC3 is a specific marker of autophagy initiation and is processed from LC3-I to LC3-II during autophagy. Then, the expression of LC3-II, as observed by immunofluorescence, can be used to track changes in autophagosome formation in osteosarcoma cells. As shown in Fig. 2b, cells treated with metformin exhibited few punctate pattern of LC3-II fluorescence, showing a reduction of LC3-II in autophagosomes. Decreased LC3II expression and an accompanying increase in p62 expression were clearly detected by western blot (Fig. 2c).

Next, we wondered whether autophagy suppression was involved in the restraining effect of metformin on the migration and the invasion of osteosarcoma. Herein, we knockdown ATG5, a crucial gene for autophagy induction, in 143B cells with siRNA and an abrogation of cell invasion and migration was observed (Fig. 2d). In addition, we demonstrated that treatment with specific autophagy inhibitor 3-methyladenine (3MA) inhibited the invasion and migration of osteosarcoma cells (Fig. 2e), suggesting that both genetic and pharmacological inhibition of autophagy suppressed the invasive and migratory ability of human osteosarcoma cells.

\section{Metformin-mediated osteosarcoma cell invasion and autophagy by negatively regulating miR-570-3p expression in osteosarcoma}

Recently, it is reported that metformin antitumor effects were proved to be connected, at least in part, with a regulation of microRNA expression ${ }^{30}$.To figure out the molecular mechanisms by which metformin affects osteosarcoma cell invasion and autophagy, we performed a miRNA microarray assay to screen for miRNAs 


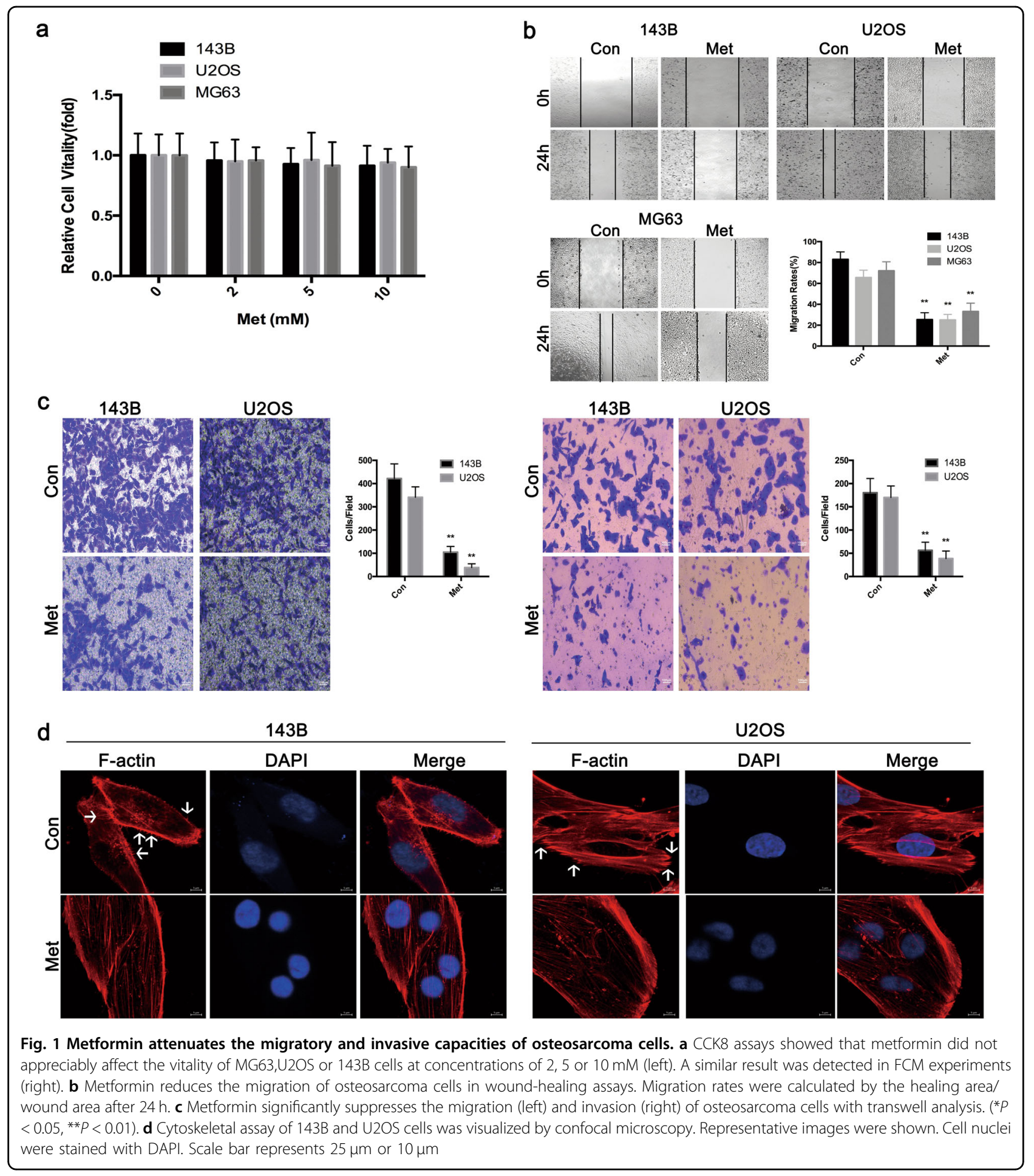

regulated by metformin. Total RNA from $143 \mathrm{~B}$ cells treated with metformin or DMSO was extracted and analyzed. We found that 23 miRNAs were upregulated, and among the changes in miRNA triggered by metformin, a marked increase in miR-570-3p was observed, which was the only one downregulated both in osteosarcoma tissues and cell lines (Fig. 3a).
Furthermore, miRNA mimics and inhibitors were used to explore the function of miR-570-3p. The expression levels of miR-570-3p were analyzed in six osteosarcoma cell lines, among which MG-63, KHOS, SAOS-2, MNNG cell lines showed significantly higher levels of miR-570-3p than U2OS and 143B cells (Fig. 3b). As demonstrated herein, in metformin-treated $143 \mathrm{~B}$ and $\mathrm{U} 2 \mathrm{OS}$ cells, there was 
a
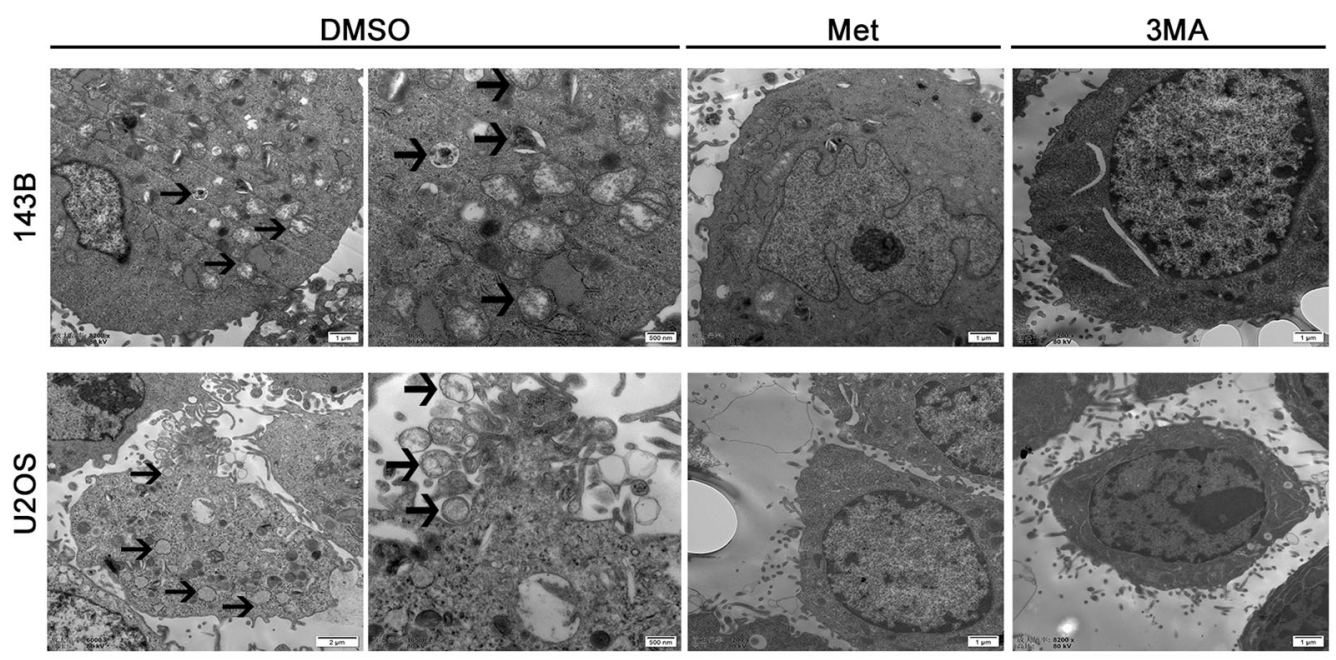

b

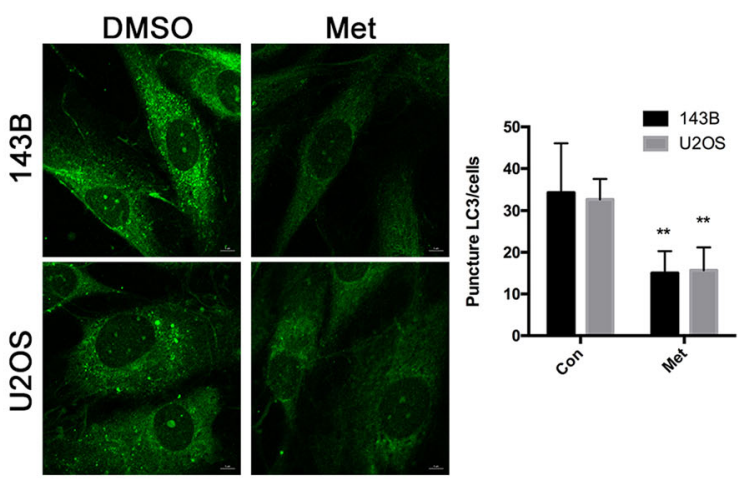

C

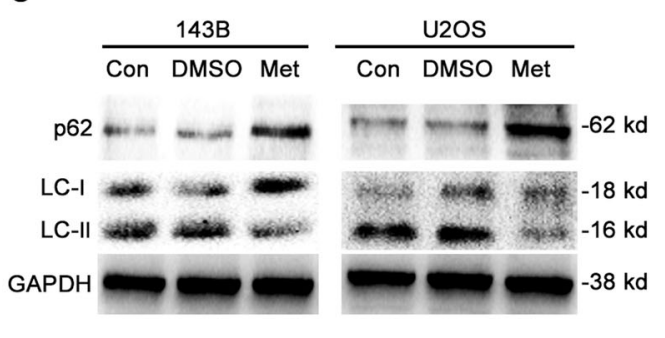

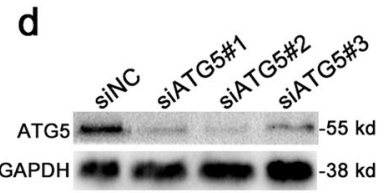

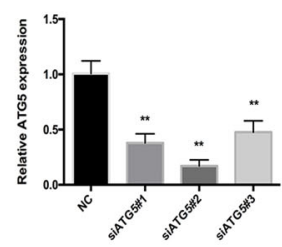

siNC
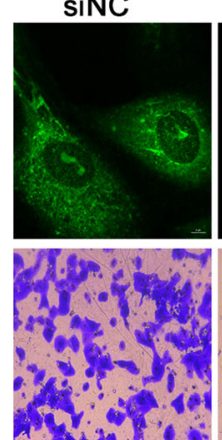

SIATG5
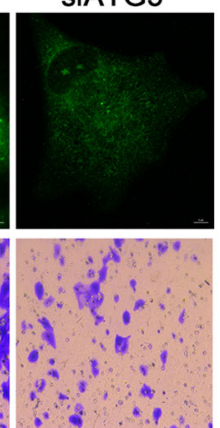

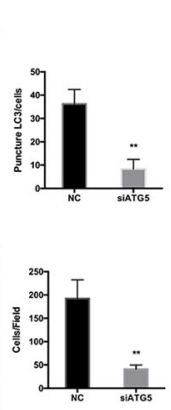

e DMSO
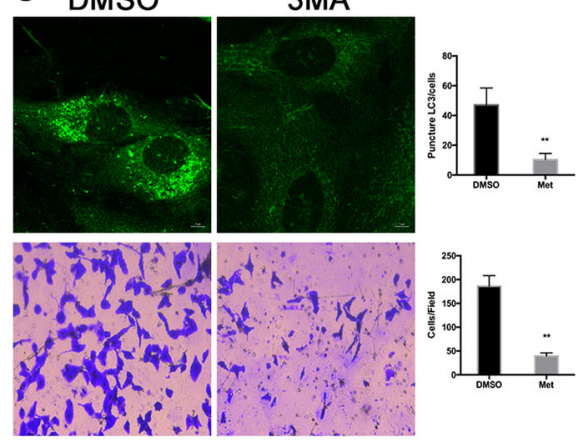

Fig. 2 Metformin-induced inhibition of autophagy indirectly suppresses osteosarcoma cell invasion. a Representative TEM images depict the ultrastructures present during autophagy in $143 \mathrm{~B}$ and U2OS cells treated with metformin or 3MA for $24 \mathrm{~h}$. The images show autophagic vacuoles (arrows) observed in control cells. No or few autophagic vacuoles were observed in metformin and 3MA-treated cells. b Cells treated with metformin exhibited a punctate pattern of LC3-II fluorescence, showing a reduction of LC3-II in autophagosomes. c Western blot analysis was used to evaluate the expression of LC3 and p62. d Suppression of autophagy by knockdown of autophagy-related gene ATG5 could inhibit invasion of osteosarcoma cells. e Osteosarcoma cells treated with autophagy inhibitor 3MA also showed significantly inhibition of invasion

increased expression of miR-570-3p (Fig. 3c). After 143B cells were transfected with anti-miR-570-3p or anti-con for $12 \mathrm{~h}$, the tumor cells were exposed to 0.0 or $10 \mathrm{mM}$ metformin for $48 \mathrm{~h}$. Metformin attenuated the invasion and autophagy, as determined by transwell assays, in osteosarcoma cells, however, the knockdown of miR-570$3 p$ decreased these effects in osteosarcoma cells (Fig. 3d).

These data suggest that miR-570-3p has a significant role in the metformin-induced inhibition of invasion and autophagy in human osteosarcoma. 


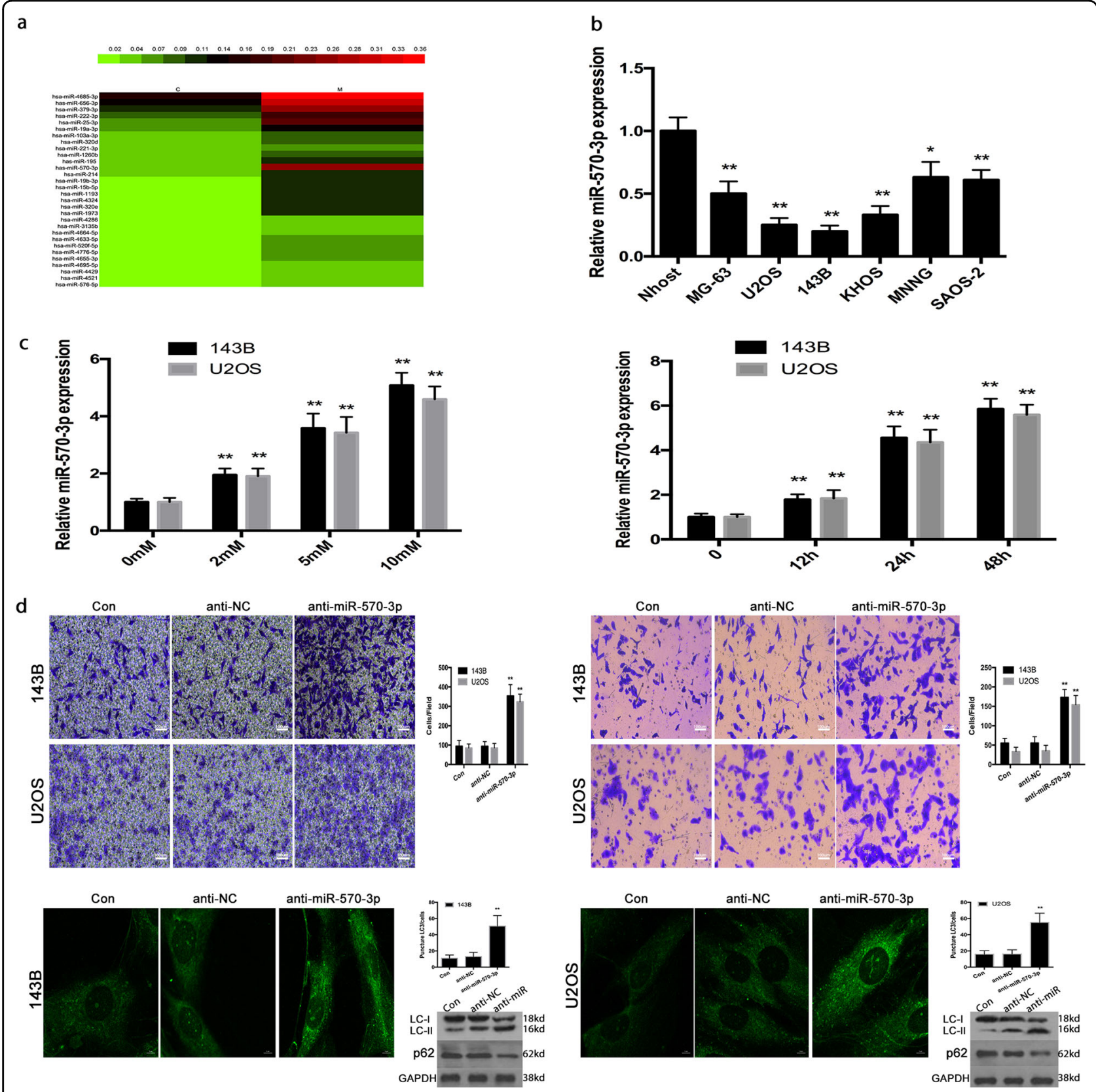

Fig. 3 Metformin-mediated osteosarcoma cell invasion and autophagy by negatively regulating miR-570-3p expression in osteosarcoma. a Heat map of genes exhibiting significant induction (red) at $24 \mathrm{~h}$ after metformin treatment in 143B cells (expressed as a ratio to $143 \mathrm{~B}$ cells treated with DMSO; fold change 42, P-value 0.05, data are log2 transformed). b The expression levels of miR-570-3p were examined in six osteosarcoma cell lines. c qRT-PCR demonstrated that miR-570-3p expression was markedly enhanced after metformin treatment in a dose- and time-dependent manner. $\mathbf{d}$ The knockdown of miR-570-3p reduced the metformin effects on invasion and autophagy in osteosarcoma cells. Data represent the mean \pm S.D. $(n=3)$ in $(\mathbf{b})$ and $(\mathbf{c}){ }^{*}{ }^{*} P<0.01$ by Student's $t$-test

MiR-570-3p expression was down-regulated in human metastatic osteosarcoma tissues

The expression level of miR-570-3p was quantified by qPCR in primary non-metastatic osteosarcoma tissues, metastatic osteosarcoma tissues and osteosarcoma cell lines. The expression level of miR-570-3p was markedly lower in metastatic osteosarcoma tissues than that in nonmetastatic osteosarcoma tissues (Fig. 4a).

Next, we examined the correlation between miR-570-3p expression and osteosarcoma patient prognosis. Kaplan-Meier survival analysis displayed that the overall survival time of patients with low miR-570-3p expression 

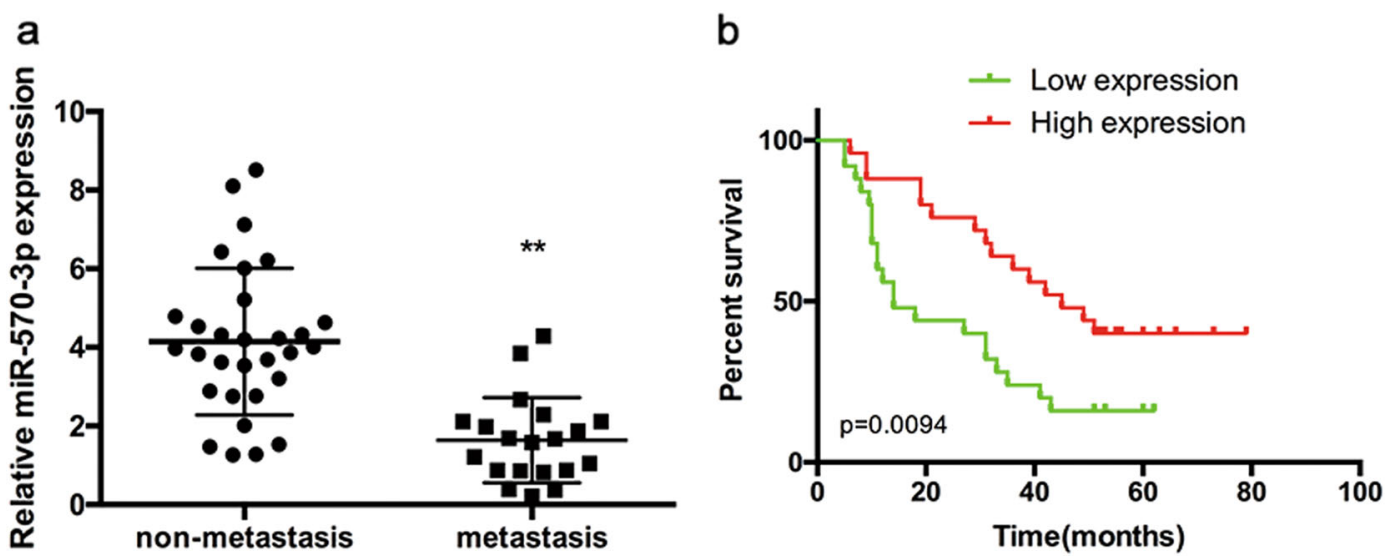

Fig. 4 MiR-570-3p expression in human osteosarcoma tissues and its relation to patient survival. a The expression of miR-570-3p was significantly lower in metastatic osteosarcoma tissues than that in non-metastatic osteosarcoma tissues. b Overall survival of 45 osteosarcoma patients. The bars illustrated S.E.M. and the significant differences between samples were analyzed using Student's $t$-test in B. All experiments were performed in three biological repeats

was observably shorter than that of patients with high miR-570-3p expression (Fig. 4b).

\section{Metformin up-regulates the expression of miR-570-3p by the demethylation of DNA in 143B and U2OS cells}

Many studies have shown that metformin could cause demethylation of DNA and lead to up-regulation of some encoding genes and non-coding RNAs ${ }^{25,26}$. We hypothesized that metformin up-regulated the expression of miR$570-3 p$ by demethylation. Bisulfate sequencing was used to analyze DNA methylation levels at the $\mathrm{CpG}$ islands of miR-570-3p promoter regions. The primer and CpG islands were shown in Fig. 5a. The results demonstrated that treatment with metformin in 143B and U2OS cells significantly decreased DNA methylation levels at the CpG islands of miR-570-3p promoter regions (Fig. 5b). Taken together, these data indicated that metformininduced demethylation of miR-570-3p promoter may, at least in part, conduce to upregulation of miR-570-3p in osteosarcoma cells.

LCMR1 and ATG12 are targets of miR-570-3p and initiators of metformin-induced metastasis inhibition and autophagy reduction

We explored the molecular mechanisms by which miR570-3p affects metformin-mediated metastasis and autophagy suppression.

As miRNAs function by targeting mRNAs, bioinformatics analyses using the target prediction tools miRNA, TargetScan and PicTar were used to identify potential binding sites in the 3'-UTRs of LCMR1 and ATG12 (Fig. 6a). We detected direct binding of miR-570-3p to the 3'-UTRs of LCMR1 and ATG12 using a dual-luciferase reporter assay (Fig. 6b) and observed significant down- regulation of LCMR1 and ATG12 in miR-570-3poverexpressing 143B cells via western blot assay and FCM (Fig. 6c).

We also found that miR-570-3p knockdown upregulated LCMR1 and ATG12 expression (Fig. 6d). In addition, we examined the effects of metformin on the expression of LCMR1 and ATG12 in osteosarcoma cells. As shown in Fig. 6e, LCMR1 and ATG12 were suppressed by metformin in a time and dose-dependent manner in 143B cells. Knockdown of miR-570-3p blocked the metformin-induced inactivation of LCMR1 and ATG12 in 143B and U2OS cells (Fig. 6f). Furthermore, over-expressing ATG12 and/or LCMR1 prevents effects of metformin and miR-570 on migration and invasion (Figure S1). Additionally, we analyzed the expression of LCMR1 and ATG12 in metastatic and primary non-metastatic osteosarcoma tissues. Our western blot assay suggested that LCMR1 and ATG12 expression was notably increased in metastatic osteosarcoma compared with the primary non-metastatic ones (Fig. 6g).

\section{Metformin up-regulates the expression of miR-570-3p and inhibits metastasis in vivo \\ To further verify these in vitro findings, we used an} in vivo xenograft model. After 143B cells were injected intravenously into the tail vein at 2 weeks, the mice were intraperitoneally administered metformin (0 or $250 \mathrm{mg} / \mathrm{kg}$ body weight) in $100 \mu \mathrm{l}$ of sterile saline every second day for another 30 days. The metformin-treated group exhibited statistically notably fewer numbers of lung metastases than the control group. Hematoxylin and eosin (HE) staining showed that fewer lung metastatic nodes were detected in the group treated with metformin (Fig. 7d). 

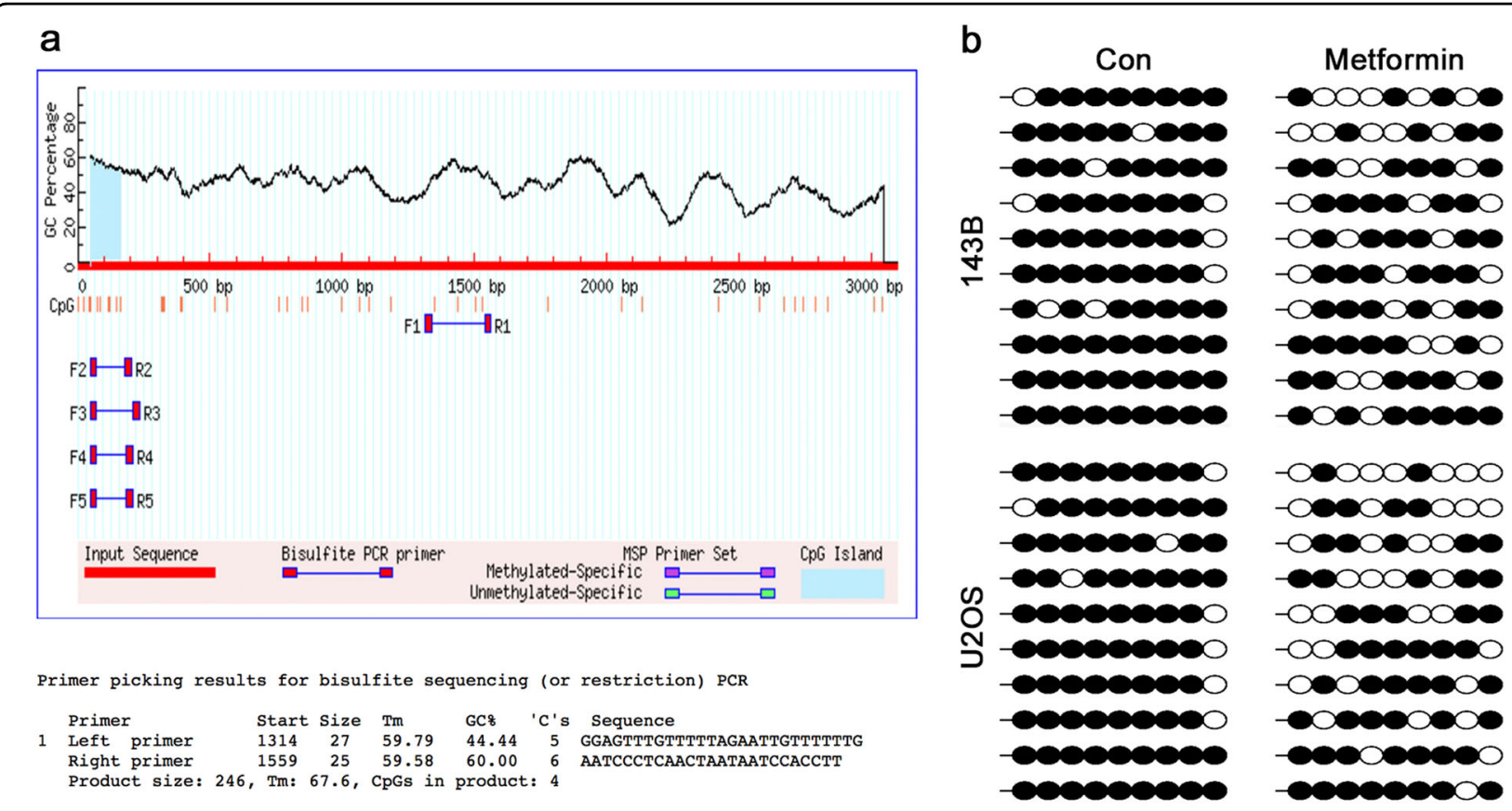

Fig. 5 Metformin up-regulates the expression of miR-570-3p by the demethylation of DNA in $143 B$ and U2OS cells. a The CpG islands of miR-570-3p promoter regions and primer used for DNA methylation study. b Metformin treatment in 143B and U2OS cells significantly decreased DNA methylation levels at the CpG islands of miR-570-3p promoter regions

To validate the function of miR-570-3p in metformininduced inhibition of metastasis, we established stable anti-con and anti-miR-570-3p 143B cell lines. Two weeks later, after the two stable cell lines were injected intravenously into the tail vein, the mice were treated as previously described. Compared to the anti-miR-570-3p group, fewer lung metastatic nodules were found in the anti-con group (Fig. 7e).The xenograft tumors were removed and evaluated by qRT-PCR, immunohistochemistry and WB (Fig. 7a-c).

\section{Discussion}

Osteosarcoma is the most common primary malignant bone tumor. The cure rate of patients of osteosarcoma is still very low because of high aggressiveness with rapid development of distant metastasis.

Metformin is the most widely used drug for therapy of type II diabetes. Epidemiological studies have demonstrated that metformin could mitigate the progression of several tumors ${ }^{24-27}$. The earliest function of metformin is to reduce blood glucose levels through abating hepatic gluconeogenesis and increasing glucose uptake in the skeletal muscles ${ }^{31}$. It has been reported that metformin treatment results in activation of AMPK in vitro and in vivo ${ }^{32-34}$. Some studies have shown that metformin metabolic action leaded to a direct and broad regulation of some miRNAs that involved in the progression of tumors in vitro and in vivo ${ }^{35,36}$.
There is little known about the effects of metformin on metastasis, and the interaction between metastasis and autophagy in human osteosarcoma. Many investigations have implicated diverse mechanisms underlying the antitumor action of metformin, with different mechanisms playing important roles in different tissues and in different cancer types ${ }^{37,38}$. This difference may be related with tissue specificity. In the present study we found that a low concentration of metformin $(10 \mathrm{mM})$ attenuates the metastasis and autophagy in osteosarcoma. Interestingly, this autophagy favors osteosarcoma cells invasion. Moreover, reduction of metformin-induced inhibition of autophagy could reverse the invasion suppression in osteosarcoma. Our data verified, for the first time, that autophagy regulates metastasis in osteosarcoma.

However, the mechanism about inhibition of metastasis and autophagy by metformin is obscure in osteosarcoma. MiRNAs are small non-coding RNAs that modulate gene expression by binding to the $3^{\prime}$-UTR of the target mRNA and suppressing translation or targeting the mRNA for degradation ${ }^{12}$. Almost all kinds of tumors, including osteosarcoma, display specific profile of aberrant miRNAs expression $^{39-48}$.

To elucidate the molecular mechanism underlying metformin function, a miRNA microarray assay and bisulfite sequencing analysis (BSP) were performed. Herein, in metformin-treated human osteosarcoma cells, for the first time, we identified miR-570-3p as a tumor 

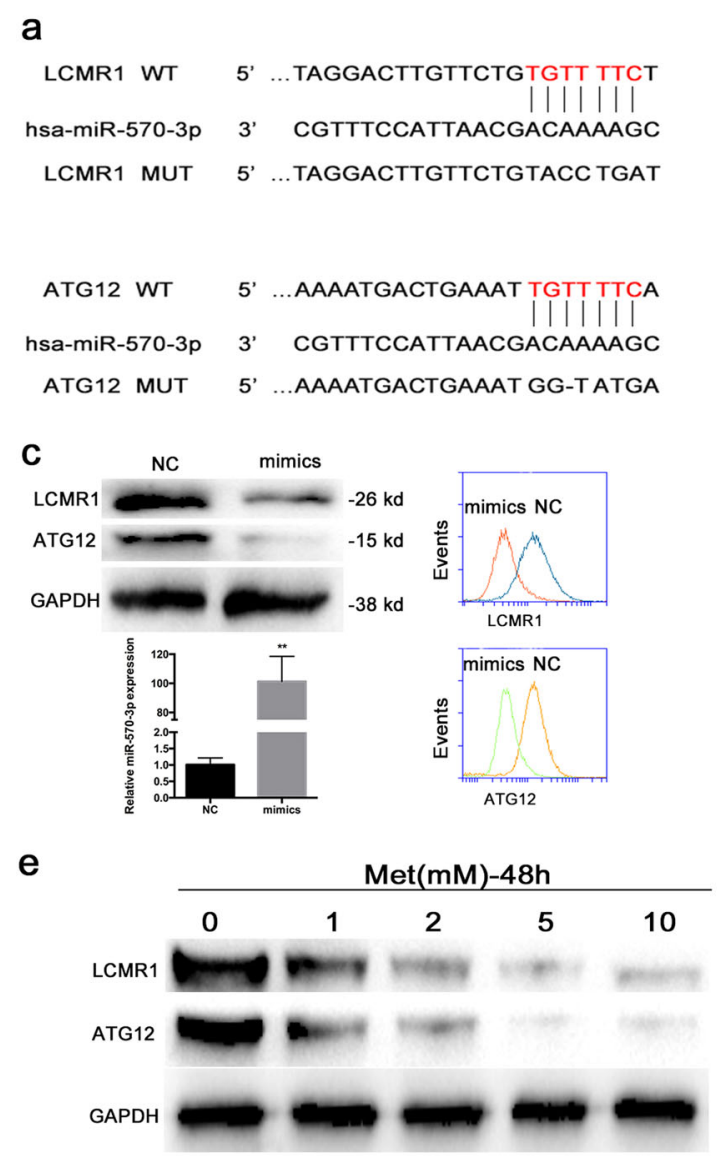

$\mathbf{f}$
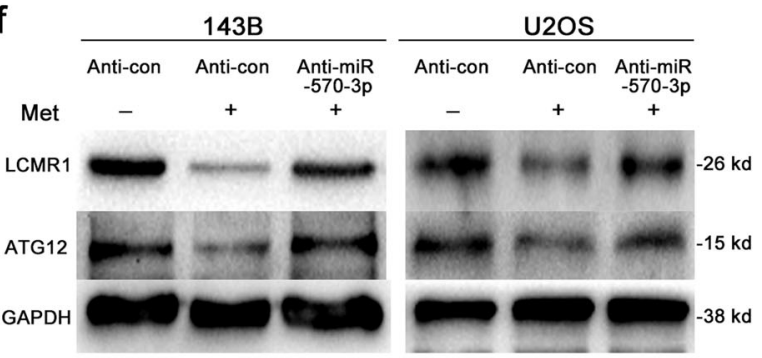

b
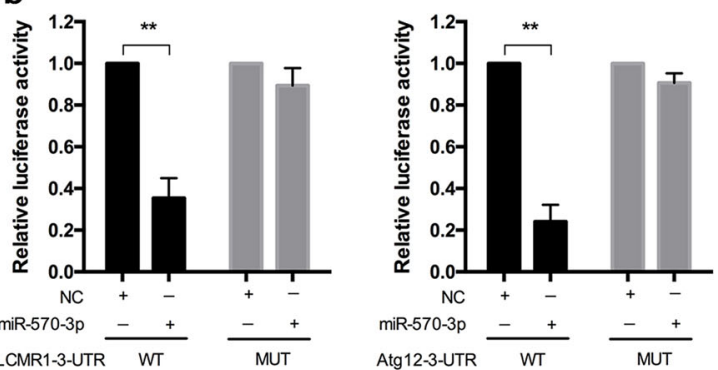

d
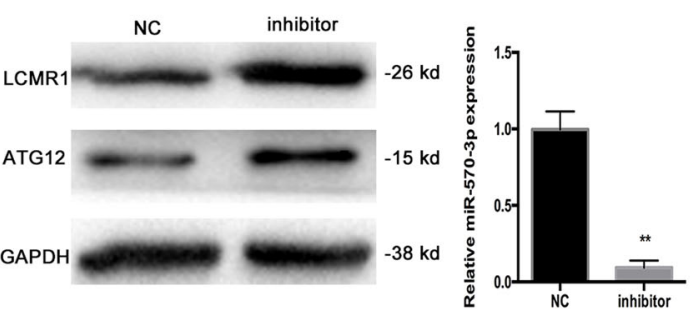

g

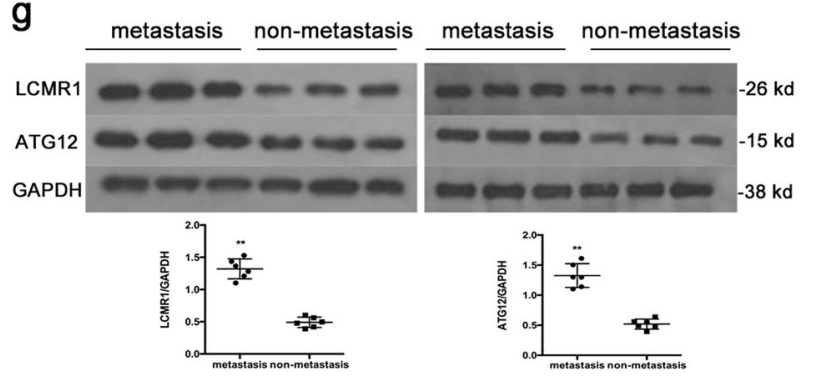

Fig. 6 LCMR1 and Atg12 are targets of miR-570-3p and initiators of metformin-induced metastasis inhibition and autophagy reduction. a The LCMR1 and Atg12 3'UTR region containing the wild type or mutant binding site for miR-570-3p. b Dual-luciferase assays were performed in 143B cells after co-transfection with wild-type or mutant LCMR1 and Atg12 3'-UTR plasmids and with NC or miR-570-3p mimics. c Western blot and FCM demonstrated the significant downregulation of LCMR1 and Atg12 in miR-570-3p-overexpressing cells compared with NC cells. d Upregulation of LCMR1 and Atg12 in miR-570-3p knockdown cells. e Metformin inhibits LCMR1 and Atg12 in a time and dose-dependent manner. $\mathbf{f}$ Knockdown of miR-570-3p blocked the metformin-induced inactivation of LCMR1 and Atg12 in 143B and U2OS cells. $\mathbf{g}$ The expression of LCMR1 and Atg12 in metastatic and primary non-metastatic osteosarcoma tissues

suppressor gene, which inhibited the metastasis and autophagy by targeting LCMR1(lung cancer metastasisrelated protein), known as Med19 in human, which is crucial in the metastasis of various human tumors ${ }^{49-51}$, and another gene, autophagy-related gene 12 (ATG12), which is an autophagy marker that is significant for the development of many types of tumors.
MiR-570-3p has been reported to be dysregulated in various tumors ${ }^{52,53}$. According to our data, the expression level of miR-570-3p was significantly lower in metastatic osteosarcoma tissues than that in non-metastatic osteosarcoma tissues. We also investigated the methylation status of those sequences in metastatic and non-metastatic tumor tissues (Figure S2). We further validated that metformin 


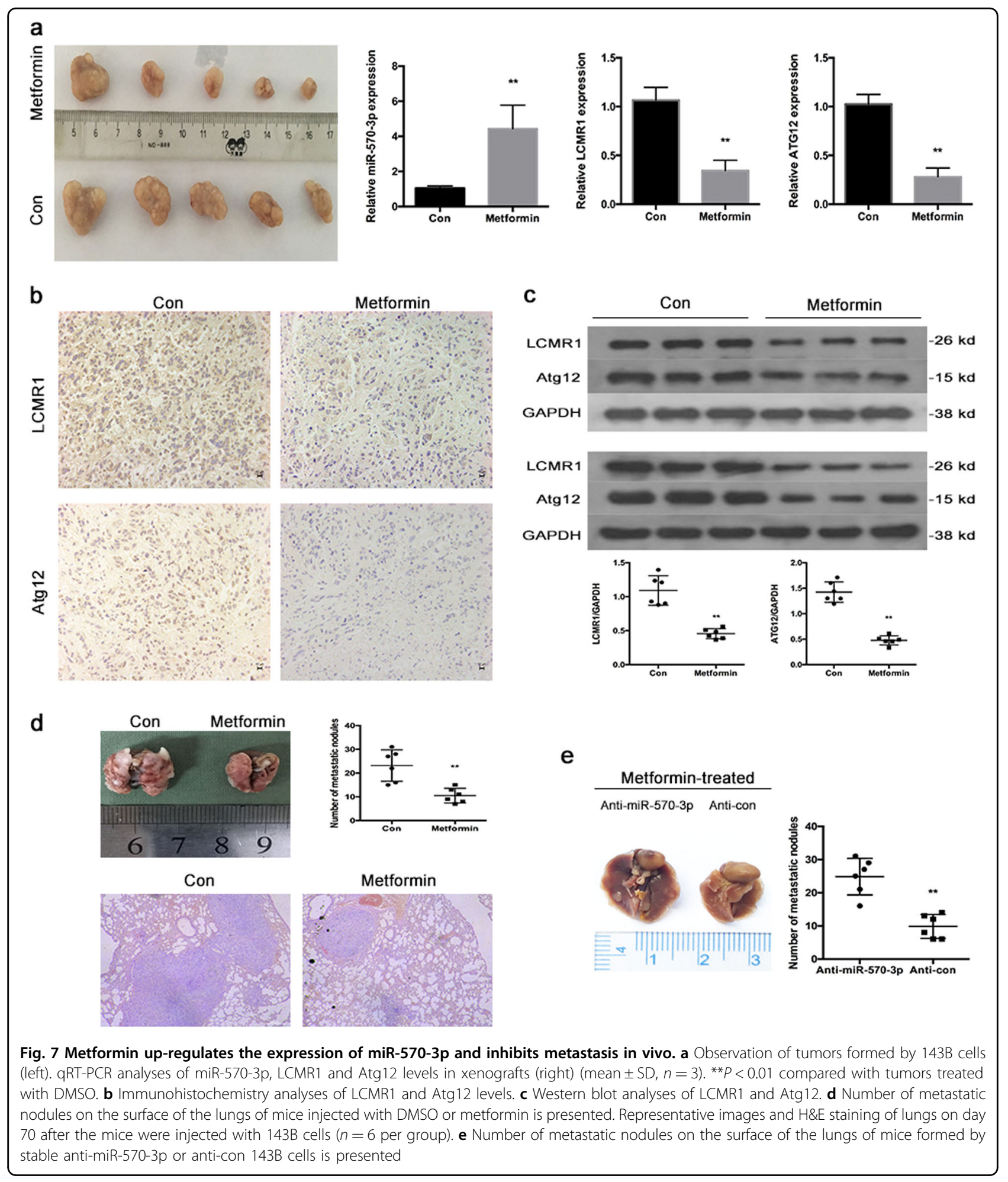

increases the miR-570-3p expression by changing the DNA methylation level of miR-570-3p promoter regions.

Overall, this study reveals a promising therapeutics target in osteosarcoma and probably in other kinds of metastatic malignant tumors. We also propose a novel mechanism in which metformin demethylation-activate miR-570-3p and subsequently represses LCMR1 and ATG12 to inhibit metastasis and autophagy.In our study, 
Table 1 The relationship between miR-570-3p expression and clinicopathological variables of osteosarcoma

\begin{tabular}{llll}
\hline Items & \multicolumn{2}{c}{ miR-570-3p } & P-value \\
\cline { 2 - 3 } & Low & High & \\
\hline All cases & 25 & 25 & \\
Age & & & 0.3157 \\
$>18$ & 8 & 11 & \\
$<18$ & 17 & 14 & 0.1816 \\
Gender & & & \\
Male & 15 & 18 & 0.2352 \\
Female & 10 & 7 & \\
Anatomical location & & & \\
Limb bone & 13 & 19 & \\
Axial bone & 12 & 6 & \\
Metastasis & & & \\
Present & 13 & 7 & \\
Absent & 12 & & \\
\hline
\end{tabular}

we confirmed that the autophagy inhibition induced by metformin facilitated the suppression of invasion and metastasis, supporting the pro-metastatic role of autophagy in osteosarcoma. In addition, our findings suggest that miR-570-3p is a potential biomarker for predicting osteosarcoma metastasis and prognosis.

\section{Materials and methods}

\section{Clinical specimens}

Fifty osteosarcoma tissues were collected under the protocols approved by the ethics committee of Tongji Hospital. None of the patients received antitumor treatment before surgery. Informed consents (written in the light of the ethical guidelines) were obtained from all of the patients. The clinical characteristics of these patients were displayed in Table 1. Fresh tissues were stored in liquid nitrogen before RNA extraction. Clinical and histopathologic information was recorded through a retrospective review of patient records.

\section{Cell culture and reagents}

Normal human osteoblasts (NHOST) were obtained from Cambrex Bio Science and maintained in Osteoblast Growth Media (Lonza). The six human osteosarcoma cell lines MG63, U2OS, 143B, KHOS, MNNG, and SAOS2 cells were purchased from the American Type Culture Collection (ATCC). 143B, MNNG, and SAOS2 cells were cultured in Dulbecco's modified Eagle's medium (DMEM) (Hyclone, Logan, UT,USA) supplemented with $10 \%$ fetal calf serum (Gibco) at $37^{\circ} \mathrm{C}$ in a humidified atmosphere with $5 \% \mathrm{CO}_{2}$. KHOS and U2OS cells were cultured with RPMI 1640 medium (Invitrogen) supplemented with 10\% fetal calf serum (Gibco). Cells lines were cultured at $37^{\circ} \mathrm{C}$ with $5 \% \mathrm{CO}_{2}$.

3MA (M9281) were purchased from Sigma Chemical Co. (St. Louis, MO,USA). These following antibodies were used in the experiments: anti-ATG5(9980), anti-LC3 (4108), anti-p62(23214) and anti-GAPDH(2118) were from Cell Signaling Technology (Beverly, MA, USA). Anti-LCMR1 (ab179735), and anti-ATG12 (ab155589) was from Abcam (Cambridge, MA, USA).

\section{Transfection}

SiATG5 and scrambled negative control siRNA (siNC) were synthesized in GenePharma (Suzhou, China). The sequences targeting ATG5 are listed in Supplementary Table S1. MiRNA mimics and inhibitors were purchased from RiboBio(Guangzhou, China). RNAs were transfected into tumor cells using Lipofectamine3000 (Invitrogen). MiR-570-3p and anti-miR-570-3p stably expressed osteosarcoma cells were infected with the lentivirus and selected with puromycin $(1 \mu \mathrm{g} / \mathrm{ml})$ for 4 weeks.

\section{CCK-8 assay}

Cells were plated in 96-well plates at a density of 5000 cells in $100 \mu \mathrm{L}$ medium per well 1 day before the experiment. The cell viability was examined by CCK-8 kit (Dojindo Laboratories, Kumamoto, Japan) according to the manufacturer's instruction.

\section{Wound healing assay}

A total of $2 \times 10^{5}$ osteosarcoma cells were seeded into a 24-well plate. The tumor cells were grown to confluence $24 \mathrm{~h}$ later. An artificial wound was introduced with a P10 pipette tip in each well. The data of the wounded area were recorded at $0 \mathrm{~h}$ and $24 \mathrm{~h}$ with a microscope (Olympus Corp). The entire assay was repeated three times.

\section{Transwell assay}

143B and U2OS cells were harvested, washed, suspended with RPMI1640 or DMEM medium, and seeded into the upper chambers of transwell inserts $(8 \mu \mathrm{m}$ pore size; Corning) with or without $10 \mathrm{mM}$ Metformin in the migration assay. The upper chambers were coated with Matrigel (BD Bioscience, 354234) before the inoculation of the cancer cells and Metformin in the invasion assay.

The lower compartments were filled with RPMI1640 or DMEM medium supplemented with 5\% FBS (fetal bovine serum). The cells in the upper chamber were removed with a swab after incubation for $12 \mathrm{~h}$ in the migration assay or $24 \mathrm{~h}$ in the invasion assay. The cells that migrated to the lower layer and attached to the membrane were stained with crystal violet and were counted in five fields 
per well under a microscope. The whole assay was repeated three times.

\section{Western blot analysis}

Equal amounts of proteins collected from different kinds of cell lysates were loaded on 10-15\% SDS-PAGE gels using a NuPAGE system (Invitrogen) and then transferred onto PVDF membranes as previously described $^{22}$.

\section{Flow cytometry (FCM) experiments}

Cells were fixed in 70\% ethanol, digested with RNase A. Fluorescence-activated cell sorting was performed according to the manufacturer's instructions. LCMR1 and ATG12-stained cells were quantified using flow cytometry as previously described ${ }^{25}$.

\section{Immunohistochemistry and immunofluorescence assay}

IHC staining was performed as previously described ${ }^{54}$. Paraffin sections were reacted with rabbit polyclonal antiLCMR1 and anti-ATG12 antibodies (1:100 dilution). Sections stained with non-immune rabbit serum (1:200 dilution) in phosphate-buffered saline (PBS) instead of primary antibody served as negative controls. Cells displaying positive staining were counted in at least 12 representative fields and the mean percentage of positive cells was calculated. Immunostaining was assessed by two independent pathologists blinded to clinical characteristics and outcomes.

For immunofluorescence assay of LC3, fixed cells were permeabilized with $0.1 \%$ Triton X-100 at room temperature for $15 \mathrm{~min}$ and incubated with anti-LC3 antibody overnight at $4{ }^{\circ} \mathrm{C}$. Cells were washed 3 times with PBST (phosphate-buffered saline with Tween-20) and then incubated for $1 \mathrm{~h}$ with Cy3-conjugated goat anti-rabbit IgG at room temperature ${ }^{27}$. For immunofluorescence assay of cytoskeletal, cells $\left(2 \times 10^{5}\right.$ cells per well $)$ were adhered to coverslips in six-well plates. Cells were then fixed in 4\% paraformaldehyde for $20 \mathrm{~min}$ and incubated in PBS with $0.1 \%$ Triton X-100 for 5 min. Coverslips were then moved to a piece of parafilm in a humid chamber to which $200 \mu \mathrm{l}$ of $100 \mathrm{nM}$ rhodamine phalloidin was added (Cytoskeleton Inc. Denver, USA). They were then incubated at room temperature shielded from light for $45 \mathrm{~min}$. Nuclei were stained with PBS with $2 \mu \mathrm{g} / \mathrm{ml}$ DAPI for 4 min. The cells were then analyzed using confocal microscopy (FV10i, Olympus, Tokyo, Japan).

\section{Transmission electron microscopy (TEM)}

After $48 \mathrm{~h}$ of metformin treatment, TEM assay was performed on cells. For TEM assay, cells were digested with $0.25 \%$ trypsin and suspended at a concentration of $1.0 \times 10^{6}$ per ml and fixation was carried out at $4{ }^{\circ} \mathrm{C}$ for $6 \mathrm{~h}$ with $1.5 \%$ glutaraldehyde. Later, ultrathin sections (100 $\mathrm{nm}$ ) were prepared, stained with uranyl acetate and lead citrate and examined under an electron transmission microscope (H-600; Hitachi, Tokyo, Japan $)^{54}$.

\section{Quantitative RT-PCR (qRT-PCR)}

The total RNA was extracted by Trizol reagent (Invitrogen). The cDNAs were synthesized using a RevertAidTM First Strand cDNA Synthesis kit (Fermentas, Vilnius, Lithuania), and real-time quantitative PCR was carried out using the SYBR-Green PCR Master Mix (Applied Biosystems, Foster City, CA, USA) on a 7900 Real-Time PCR System (Applied Biosystems). MiRNA qRT-PCR Primer were purchased from RiboBio. Other genes' primer sequences are listed in Supplementary Table S1. U6 or GAPDH were used as endogenous controls. The reactions were performed in a 96-well optical plate(Applied Biosystems, Warrington, UK) at $94{ }^{\circ} \mathrm{C}$ for 2 min, followed by 38 cycles of $94^{\circ} \mathrm{C}$ for $45 \mathrm{~s}, 56^{\circ} \mathrm{C}$ for $45 \mathrm{~s}$, and $72{ }^{\circ} \mathrm{C}$ for $40 \mathrm{~s}$.

\section{MiRNA microarray assay}

Total RNA from cells treated with metformin for $24 \mathrm{~h}$ was extracted using RNeasy mini kit (Qiagen, Venlo, The Netherlands), and reverse transcribed according to the manufacturer's instructions (Fermentas, in CA). The miRNA microarray assay was performed by a commercial company (Phalanx Biotech Group, Hsinchu, Taiwan) using Human v7.1 miRNA OneArray platform that is designed to contain $100 \%$ of miRBase 21 database. Briefly, RNA was extracted using miRNA isolation kits (Qiagen ${ }^{\circledR}$ ) according to the manufacturer's protocol. RNA purified was quantified at OD $260 \mathrm{~nm}$ by an ND-1000 spectrophotometer (NanoDrop Technologies) and analyzed by the Bioanalyzer 2100 (Agilent Technologies, Santa Clara, CA, USA) with the RNA 6000 Nano LabChip kit. During the in vitro transcription process, $1 \mu \mathrm{g}$ of total RNA was amplified by a low RNA input fluor linear amp kit (Agilent) and labeled with Cy3 (CyDye, PerkinElmer, Waltham, MA, USA). Using incubation with fragmentation buffer at $60{ }^{\circ} \mathrm{C}$ for $30 \mathrm{~min}, 1.65 \mu \mathrm{g}$ of Cy3-labled cRNA was fragmented to an average size of about 50-100 nucleotides. Correspondingly fragmented labeled cRNA was then pooled and hybridized to SurePrint G3 ChIP/ $\mathrm{CH} 31 \times 1 \mathrm{M}$ array (Agilent) at $60^{\circ} \mathrm{C}$ for $17 \mathrm{~h}$. After washing and drying by nitrogen gun blowing, the microarrays were scanned with an Agilent microarray scanner at $535 \mathrm{~nm}$ for Cy3. Scanned images were analyzed by Agilent Feature Extraction, version 10.5. Image analysis and normalization software were used to quantify the signal and background intensity for each feature.

\section{Luciferase reporter assay}

The LCMR1 and ATG12 3'-untranslated region (3'UTR) containing the wild type or mutated miR-570-3p 
binding sequences were synthesized by Genescript (Nanjing, Jiangsu,China), and were cloned into the pmirGLO luciferase reporter vector (Promega, Madison, WI, USA). HEK293 cells were transfected with the wild type/mutant LCMR1 and ATG12 luciferase reporter vector and miR-570-3p mimic/miR-Control using Lipofectamine 3000. Firefly and Renilla luciferase activities were measured using the Dual-Luciferase Reporter Assay System (Promega). Results were expressed as the firefly luciferase activity normalized to Renilla luciferase activity.

\section{Bisulfite sequencing analysis (BSP)}

The methylation levels of miR-570-3p promoter was analyzed by BSP. miR-570-3p DNA was extracted using a DNA kit (Qiagen 51306, Germany), and $2 \mu \mathrm{g}$ of DNA was subjected to bisulfite conversion using an EpiTect Bisulfite Kit (59104, Qiagen, Germany) according to the manufacturer's instructions. The transformed DNA was then PCR-amplified using the TaKaRa rTaq Kit (R001B, TaKaRa, Dalian, China). The PCR amplification products were sequenced by Invitrogen Corporation, Shanghai.

\section{Generation of xenografts}

Six-week-old BALB/c female athymic nude mice (Vitalriver, Beijing, China) were subcutaneously injected in the right flank with osteosarcoma cells $\left(2 \times 10^{6}\right.$ in 100 $\mu \mathrm{l}$ PBS). The volume of xenografts was measured every five days $\left(\right.$ tumor volume $=\left(\right.$ length $\times$ width $\left.\left.^{2}\right) / 2\right)$. The mice were sacrificed 30 days later. Tumor samples were processed for IHC.

The tumor metastatic capacity of $143 \mathrm{~B}$ cells $\left(5 \times 10^{6}\right.$ cells) was detected following cell injection intravenously into the tail vein of mice. Four weeks later, the mice were divided into two groups at random and administered intraperitoneally with DMSO or metformin at a dose of $250 \mathrm{mg} / \mathrm{kg}$ every second day for 30 days ( $n=6$ per group); the mice were then sacrificed, and the number of lung metastatic nodules was counted. Metastatic lungs were fixed with $4 \%$ paraformaldehyde before dehydration and paraffin embedding. Paraffin sections were stained with hematoxylin and eosin according to standard protocols ${ }^{55}$.

\section{Statistical analysis}

Data are expressed as the mean \pm SEM of at least three independent experiments, and statistical evaluation was performed using one-way analysis of variance (ANOVA) or Student's $t$-tests. Values of $P<0.05$ were considered statistically significant.

\section{Acknowledgements}

This research was supported by the National Nature Science Foundation of China (Nos. 81572633; 81472509).

\section{Authors' contributions}

X.B. and L.Z. are co-first authors. X.B. and F.L. conceived and designed the study. X.B. and L.Z. performed the experiments. X.B. analyzed and interpreted the data. H.G. and L.Z. contributed to materials. X.B. wrote the manuscript. All authors read and approved the final manuscript.

\section{Conflict of interest}

The authors declare that they have no conflict of interest.

\section{Publisher's note}

Springer Nature remains neutral with regard to jurisdictional claims in published maps and institutional affiliations.

Supplementary Information accompanies this paper at (https://doi.org/ 10.1038/s41419-018-0620-z).

Received: 7 December 2017 Revised: 6 April 2018 Accepted: 20 April 2018 Published online: 23 May 2018

\section{References}

1. Luetke, A., Meyers, P. A., Lewis, I. \& Juergens, H. Osteosarcoma treatmentwhere do we stand? A state of the art review. Cancer Treat. Rev. 40, 523-532 (2014).

2. Rettew, A. N. et al. Multiple receptor tyrosine kinases promote the in vitro phenotype of metastatic human osteosarcoma cell lines. Oncogenesis 1, e34 (2012).

3. Hauben, E. I. et al. Multiple primary malignancies in osteosarcoma patients. Incidence and predictive value of osteosarcoma subtype for cancer syndromes related with osteosarcoma. Eur. J. Hum. Genet. 11, 611-618 (2003).

4. Ferguson, W. S. \& Goorin, A. M. Current treatment of osteosarcoma. Cancer Invest. 19, 292-315 (2001).

5. Geller, D. S. \& Gorlick, R. Osteosarcoma: a review of diagnosis,management and treatment strategies. Clin. Adv. Hematol. Oncol. 8, 705-718 (2010).

6. Liang, Y. J. et al. MiR-124 targets Slug to regulate epithelial-mesenchymal transition and metastasis of breast cancer. Carcinogenesis 34, 713-722 (2013).

7. Fan, Z. et al. MiR-125a suppresses tumor growth, invasion and metastasis in cervical cancer by targeting STAT3. Oncotarget 6, 25266-25280 (2015).

8. Hamam, R. et al. Circulating microRNAs in breast cancer: novel diagnostic and prognostic biomarkers. Cell Death Dis. 8, e3045 (2017).

9. Volinia, S. et al. A microRNA expression signature of human solid tumors defines cancer gene targets. Proc. Natl Acad. Sci. USA 103, 2257-2261 (2006).

10. Li, X. J., Ren, Z. J. \& Tang, J. H. MicroRNA-34a: a potential therapeutic target in human cancer. Cell Death Dis. 5, e1327 (2014).

11. Lim, L. P. et al. Microarray analysis shows that some microRNAs downregulate large numbers of target mRNAs. Nature 433, 769-773 (2005).

12. Baek, D. et al. The impact of microRNAs on protein output. Nature $\mathbf{4 5 5}, \mathbf{6 4 - 7 1}$ (2008).

13. Liang et al. The EGFR/miR-338-3p/EYA2 axis controls breast tumor growth and lung metastasis. Cell Death Dis. 8, e2928 (2017).

14. Calin, G. A. \& Croce, C. M. Chromosomal rearrangements and microRNAs: a new cancer link with clinical implications. J. Clin. Invest. 117, 2059-2066 (2007)

15. Krzeszinski, J. Y. et al. miR-34a blocks osteoporosis and bone metastasis by inhibiting osteoclastogenesis and Tgif2. Nature 512, 431-435 (2014).

16. De Veirman, $K$. et al. Induction of miR-146a by multiple myeloma cells in mesenchymal stromal cells stimulates their pro-tumoral activity. Cancer Lett. 377, 17-24 (2016)

17. Kim, C., Hong, Y., Lee, H., Kang, H. \& Lee, E. K. MicroRNA-195 desensitizes HCT116 human colon cancer cells to 5-fluorouracil. Cancer Lett. 412, 264-271 (2017).

18. Wang et al. MicroRNA-1297 inhibits the growth and metastasis of colorectal cancer by suppressing cyclin D2 expression. DNA Cell Biol. 36, 991-999 (2017).

19. Pothoulakis, C. et al. CRHR2/Ucn2 signaling is a novel regulator of miR-7/YY1/ Fas circuitry contributing to reversal of colorectal cancer cell resistance to Fasmediated apoptosis. Int. J. Cancer 142, 334-346 (2017). 
20. Croce, C. M. Causes and consequences of microRNA dysregulation in cancer. Nat. Rev. Genet. 10, 704-714 (2009).

21. Wang, $Y$. et al. Arsenic trioxide inhibits breast cancer cell growth via microRNA328/hERG pathway in MCF-7 cells. Mol. Med. Rep. 12, 1233-1238 (2015).

22. Bao, X. et al. Knockdown of long non-coding RNA HOTAIR increases miR-454$3 p$ by targeting Stat3 and Atg12 to inhibit chondrosarcoma growth. Cell Death Dis. 8, e2605 (2017).

23. Nilsson, S., Huelsenbeck, J. \& Fritz, G. Mevalonate pathway inhibitors affect anticancer drug-induced cell death and DNA damage response of human sarcoma cells. Cancer Lett. 304, 60-69 (2011).

24. Rosilio, C., Ben-Sahra, I., Bost, F. \& Peyron, J. F. Metformin: a metabolic disruptor and anti-diabetic drug to target human leukemia. Cancer Lett. 346, 188-196 (2014).

25. Zhong, T. et al. Metformin alters DNA methylation genome-wide via the H19/ SAHH axis. Oncogene 36, 2345-2354 (2017).

26. Zhou, J. et al. Regulation of tumor cell migration and invasion by the H19/let-7 axis is antagonized by metformin-induced DNA methylation. Oncogene $\mathbf{3 4}$ 3076-3084 (2015).

27. Feng, Y. et al. Metformin promotes autophagy and apoptosis in esophageal squamous cell carcinoma by downregulating Stat3 signaling. Cell Death Dis. $\mathbf{5}$, e1088 (2014).

28. Kenific, C. M. \& Debnath, J. Cellular and metabolic functions for autophagy in cancer cells. Trends Cell Biol. 25, 37-45 (2015).

29. $\mathrm{Yu}, \mathrm{X}$. et al. MiR-214 increases the sensitivity of breast cancer cells to tamoxifen and fulvestrant through inhibition of autophagy. Mol. Cancer 14, 208 (2015).

30. Noren Hooten, $\mathrm{N}$. et al. Metformin-mediated increase in DICER1 regulates microRNA expression and cellular senescence. Aging Cell. 15, 572-581 (2016).

31. Viollet, B. et al. Cellular and molecular mechanisms of metformin: an overview. Clin. Sci. 122, 253-270 (2012).

32. Stephenne, X. et al. Metformin activates AMP-activated protein kinase in primary human hepatocytes by decreasing cellular energy status. Diabetologia 54, 3101-3110 (2011).

33. Turban, S. et al. Defining the contribution of AMP-activated protein kinase (AMPK) and protein kinase $C$ (PKC) in regulation of glucose uptake by metformin in skeletal muscle cells. J. Biol. Chem. 287, 20088-20099 (2012).

34. Viollet B., Andreelli F. AMP-activated protein kinase and metabolic control. Handb. Exp. Pharmacol. 203, 303-330 (2011).

35. Wahdan-Alaswad, R. S. et al. Metformin-induced killing of triple-negative breast cancer cells is mediated by reduction in fatty acid synthase via miRNA193b. Horm. Cancer 5, 374-389 (2014).

36. Banerjee, P., Dutta, S. \& Pal, R. Dysregulation of Wnt-signaling and a candidate set of miRNAs underlie the effect of metforminon neural crest cell development. Stem Cells. 34, 334-345 (2016).

37. Shi, W. Y. et al. Therapeutic metformin/AMPK activation blocked lymphoma cell growth via inhibition of mTOR pathway and induction of autophagy. Cell Death Dis. 3, e275 (2012).

38. Tomic, T. et al. Metformin inhibits melanoma development through autophagy and apoptosis mechanisms. Cell Death Dis. 2, e199 (2011).

39. Lulla, R. R. et al. Identification of differentially expressed microRNAs in osteosarcoma. Sarcoma 2011, 732690 (2011).
40. Rodriguez Calleja, L. et al. $\triangle N p 63$ a silences a miRNA program to aberrantly initiate a wound-healing program that promotes TGF $\beta$-induced metastasis. Cancer Res. 76, 3236-3251 (2016).

41. Robin, T. P. et al. EWS/FLI1 regulates EYA3 in Ewing sarcoma via modulation of miRNA-708, resulting in increased cell survival and chemoresistance. Mol. Cancer Res. 10, 1098-1108 (2012).

42. Yoshitaka, T. et al. Analysis of microRNAs expressions in chondrosarcoma. J. Orthop. Res. 31, 1992-1998 (2013).

43. Zhu, Z., Wang, C. P., Zhang, Y. F. \& Nie, L. MicroRNA-100 resensitizes resistant chondrosarcoma cells to cisplatin through direct targeting of mTOR. Asian Pac. J. Cancer Prev. 15, 917-923 (2014).

44. Yang, C. et al. miR-1301 inhibits hepatocellular carcinoma cell migration, invasion, and angiogenesis by decreasing Wnt/ $\beta$-catenin signaling through targeting BCL9. Cell Death Dis. 8, e2999 (2017).

45. Yu, Y., Nangia-Makker, P., Farhana, L. \& Majumdar, A. P. N. A novel mechanism of IncRNA and miRNA interaction: CCAT2 regulates miR-145 expression by suppressing its maturation process in colon cancer cells. Mol. Cancer. 16, 155 (2017).

46. Zuntini, M. et al. MicroRNA profiling of multiple osteochondromas: identification of diseasespecific and normal cartilage signatures. Clin. Genet. 78, 507-516 (2010).

47. Nugent, M. microRNA and bone cancer. Adv. Exp. Med Biol. 889, 201-230 (2015).

48. Speetjens, F. M., de Jong, Y., Gelderblom, H. \& Bovée, J. V. Molecular oncogenesis of chondrosarcoma: impact for targeted treatment. Curr. Opin. Oncol. 28, 314-322 (2016).

49. Ji-Fu, E., Xing, J. J., Hao, L. Q. \& Fu, C. G. Suppression of lung cancer metastasisrelated protein 1 (LCMR1) inhibits the growth of colorectal cancer cells. Mol. Biol. Rep. 39, 3675-3681 (2012).

50. Xu, Y., Liang, Z., Li, C., Yang, Z. \& Chen, L. LCMR1 interacts with DEK to suppress apoptosis in lung cancer cells. Mol. Med. Rep. 16, 4159-4164 (2017).

51. Sato, S. et al. A set of consensus mammalian mediator subunits identified by multidimensional protein identification technology. Mol. Cell. 14, 685-691 (2004).

52. Wang, W. et al. A miR-570 binding site polymorphism in the $\mathrm{B} 7-\mathrm{H} 1$ gene is associated with the risk of gastric adenocarcinoma. Hum. Genet. 132, 641-648 (2013).

53. Gupta, A. et al. Evaluation of miR-27a, miR-181a and miR-570 genetic variants with gallbladder cancer susceptibility and treatment outcome in a North Indian population. Mol. Diagn. Ther. 19, 317-327 (2015).

54. Bao, $X$. et al. Induction of the mesenchymal to epithelial transition by demethylation-activated microRNA-125b is involved in the anti-migration/ invasion effects of arsenic trioxide on human chondrosarcoma. J. Exp. Clin. Cancer Res. 35, 129 (2016).

55. Jiao, G. et al. BMPR2 inhibition induced apoptosis and autophagy via destabilization of XIAP in human chondrosarcoma cells. Cell Death Dis. 5, e1571 (2014). 